\title{
Tingling hand: magnetic resonance imaging of median nerve pathologies within the carpal tunnel
}

\author{
Ashwini Kumari ${ }^{A, B, C, D, E, F}$, Sapna Singh ${ }^{A, B, C, D, E, F}$, Anju Garg ${ }^{A, B, C, D, E, F}$, Anjali Prakash ${ }^{A, B, D, E}$, Sumit Sural ${ }^{A, B, D, E}$ \\ Maulana Azad Medical College, New Delhi, India
}

\begin{abstract}
Compressive neuropathy of the median nerve at the level of the carpal tunnel, known as carpal tunnel syndrome, is the most common entrapment neuropathy, affecting about $0.1-1 \%$ of the general population. Magnetic resonance reliably imaged the flexor retinaculum and carpal bones and thus defined the borders of the carpal tunnel. In all cases the median nerve was seen as an ovoid structure of moderate signal intensity and was easily distinguished from the flexor tendons of the hands running in the carpal tunnel. Magnetic resonance imaging (MRI) serves as an extremely useful tool for evaluation of primary nerve pathologies and for the assessment of space-occupying lesions leading to its compression. We present a pictorial review of the MRI findings in the multitude of pathologies implicated in the causation of carpal tunnel syndrome. All the images were obtained from the Department of Radiodiagnosis in our own institution.
\end{abstract}

Key words: tuberculosis, carpal tunnel syndrome, neuroma, fibrolipomatous hamartoma, Hansen's neuritis, bifid median nerve.

\section{Introduction}

Compressive neuropathy of the median nerve at the level of the carpal tunnel, known as carpal tunnel syndrome (CTS), accounts for about $90 \%$ of all compressive neuropathies. It occurs commonly in patients between 30 and 60 years of age and has a female-to-male ratio of between 3 and $5: 1$ because of the smaller cross-sectional area of carpal tunnel in females both proximally and distally. Up to $50 \%$ of the cases are bilateral [1].

\section{Anatomy of carpal tunnel}

The carpal tunnel is a fibro-osseus tunnel at the level of the wrist joint. The deep border of the carpal tunnel is formed by the carpal bones, and the superficial border is formed by a thick ligamentous band known as the flexor retinaculum. The attachments of the flexor retinaculum are to the hook of the hamate and pisiform bone medially and the tubercle of the trapezium and scaphoid laterally. The ligament is thickest distally where it attaches to the hook of the hamate and tubercle of the trapezium. The contents include nine tendons and a nerve: flexor pollicis longus (FPL), flexor digitorum superficialis (FDS), flexor digitorum profundus (FDP), and median nerve (Figure 1).

\section{Structure of the median nerve at the level of the carpal tunnel}

The median nerve is best evaluated in the axial plane, where it appears rounded at the level of the distal radius and elliptical at the level of pisiform. Like all peripheral nerves of the body, the median nerve appears isointense to skeletal muscles on T1- and minimally hyperintense on T2-weighted images [2]. A fascicular pattern is seen on both T1 and T2 sequences (Figure 2). The course is well outlined by perineural fat and is seen best on $\mathrm{T} 1$ sequence. There is no appreciable post-contrast enhancement seen normally.

\footnotetext{
Correspondence address:

Ashwini Kumari, Maulana Azad Medical College, Bahadur Shah Zafar Marg, Maulana Azad Medical College Campus, Balmiki Basti, New Delhi, Delhi 110002,

India, e-mail: ashwini.kims@gmail.com
}

Authors' contribution:

A Study design · B Data collection · C Statistical analysis · D Data interpretation - E Manuscript preparation · F Literature search · G Funds collection 


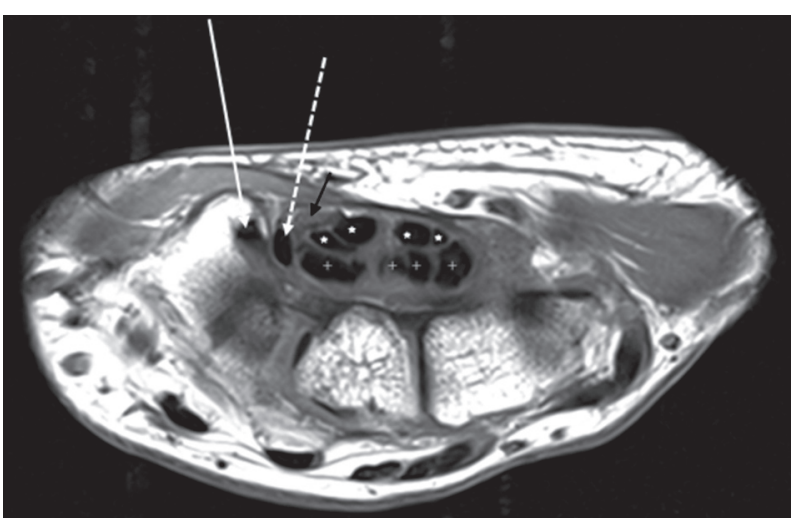

Figure 1. Axial T1-weighted image of the right wrist at the level of the tubercle of trapezium (T) shows the normal flexor tendons and median nerve located volarly. The flexor carpi radialis (white arrow) is seen to lie in a fibroosseus tunnel, situated radial and volar to the carpal tunnel. The flexor pollicis longus (dashed white arrow) is the most radially located tendon within the carpal tunnel. The flexor digitorum superficialis (white asterisk) and flexor digitorum profundus (white cross) tendons lie within the carpal tunnel. The median nerve (black arrow) appears fasciculated and shows intermediate to high signal

\section{Aetiologies of carpal tunnel syndrome}

The median nerve at the level of the carpal tunnel can be affected by various conditions, which can individually be grouped into two categories: extrinsic and intrinsic (Table 1).
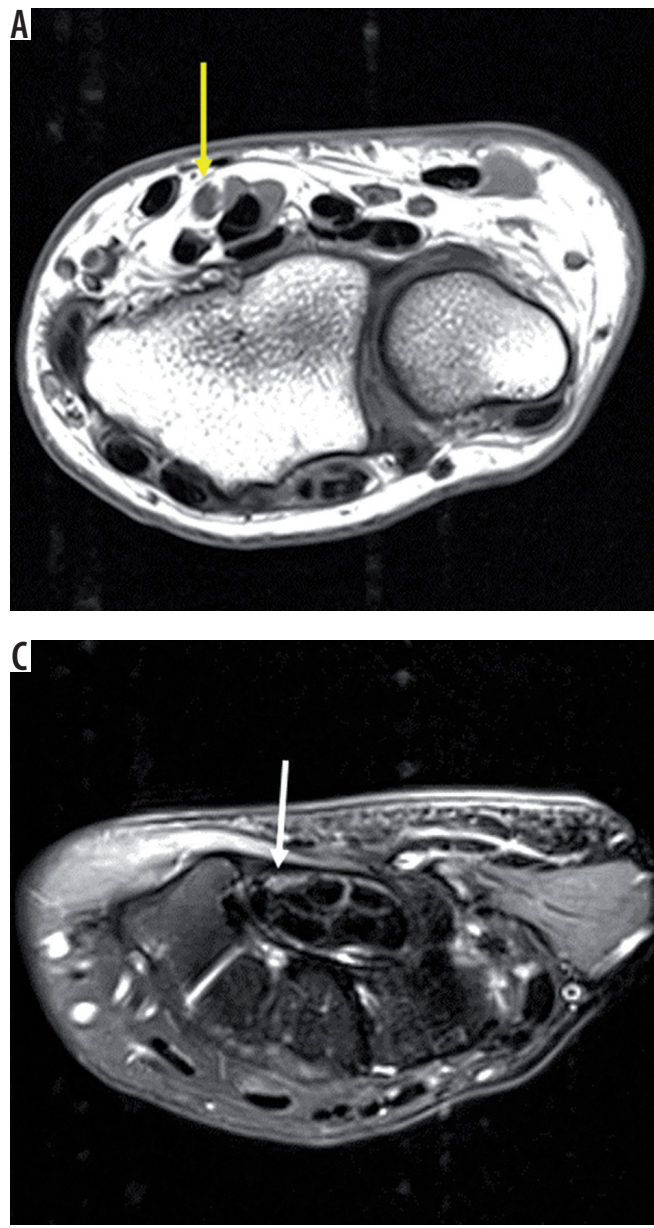

Table 1. Carpal tunnel

\begin{tabular}{|l|l|}
\hline Extrinsic & Intrinsic \\
\hline Idiopathic & Post traumatic neuroma \\
\hline Rheumatoid arthritis & $\begin{array}{l}\text { Infections causing neuritis primarily } \\
\text { (e.g. Hansen's disease) }\end{array}$ \\
\hline $\begin{array}{l}\text { Infections causing flexor } \\
\text { tenosynovitis (e.g. tuberculosis) }\end{array}$ & Fibrolipomatous hamartoma \\
\hline Trauma & $\begin{array}{l}\text { Bifid median nerve with persistent } \\
\text { median artery }\end{array}$ \\
\hline
\end{tabular}

A multitude of general conditions may also lead to carpal tunnel syndrome. The most common among them are pregnancy, diabetes mellitus, obesity, and hypothyroidism.

\section{Idiopathic carpal tunnel syndrome}

Idiopathic carpal tunnel syndrome is the most common peripheral nerve lesion [3].

The findings of CTS on magnetic resonance imaging (MRI) include (Figure 3):

I. Raised bowing ratio: First, a straight line (TH) is drawn between the attachments of the flexor retinaculum, from the tubercle of trapezium to the hook of hamate, and the length is measured. Next, a line (PD) is drawn perpendicularly from line $\mathrm{TH}$ to the palmar apex of the flexor retinaculum. Then the bowing ratio is calculated

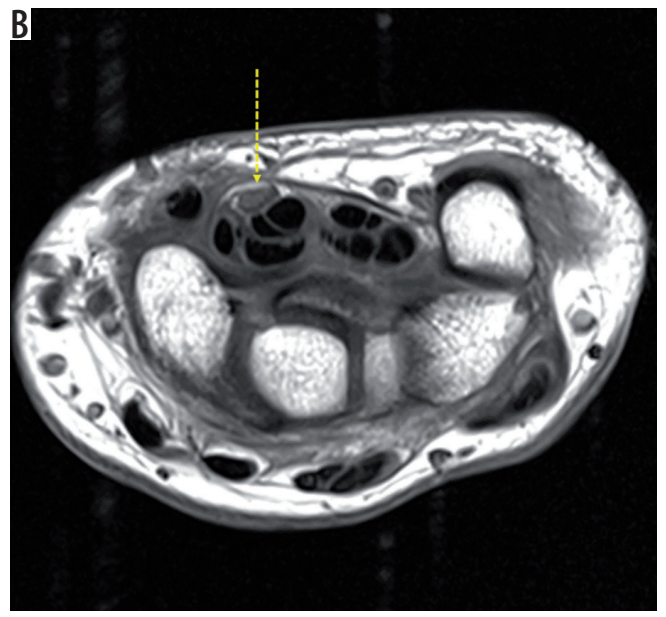

Figure 2. Axial T1-weighted images (A, B) and axial T2 fat-saturated image of the left wrist $(C)$ at the level of the distal radioulnar joint (DRUJ), pisiform $(P)$, and hamate, respectively. The median nerve appears oval at the level of the DRUJ (solid yellow arrow) and elliptical at the level of the pisiform (dashed yellow arrow) and hamate (solid white arrow). There appears to be a progressive decrease in cross-sectional area distally. The normal median nerve appears isointense to skeletal muscle on T1W and iso to minimally hyperintense on $\mathrm{T} 2 \mathrm{~W}$ images 

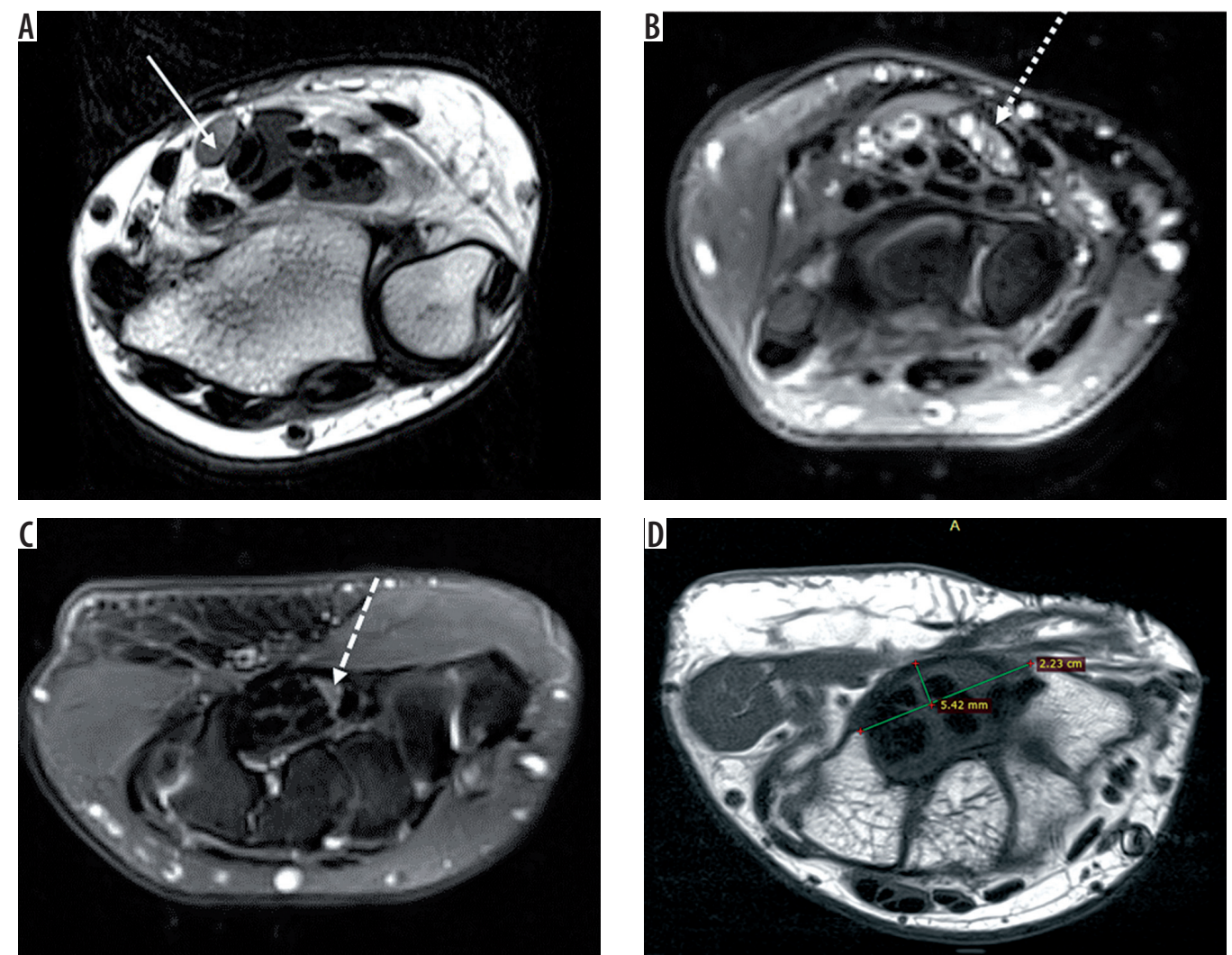

Figure 3. Axial T2-weighted image of the left wrist (A) shows bulbous enlargement of the median nerve at the level of distal radioulnar joint (solid white arrow). Axial STIR (B) image of the right wrist shows bulbous enlargement of the median nerve (dotted white arrow) at the level of the pisiform, appearing heterogeneously hyperintense. Axial STIR (C) image shows flattening and angulation of the median nerve (dashed white arrow) at the level of the hook of hamate (H). Axial T1-weighted image (D) shows palmar bowing of the flexor retinaculum with raised bowing ratio of $21^{\circ}\left(\right.$ normal $\left.<15^{\circ}\right)$. Thenar muscles show a marked decrease in bulk with $\mathrm{T} 1$ hyperintensity (black arrow) within, suggestive of fatty atrophy

as line PD divided by TH. A bowing index above 15\% is considered significant, and it correlates with findings on EMG and the patient's subjective reports of pain severity [4].

II. A difference in cross-sectional area of median nerve more than $2 \mathrm{~mm}^{2}$ between the level of the hamate and pisiform: bulbous enlargement of median nerve at the level of pisiform with flattening and angulation of the nerve at the level of hamate [5]. This occurs because the carpal tunnel is narrowest at the level of the hamate and wider proximally.

III. Hyperintense signal intensity on T2-weighted images within the median nerve [6]: Increased signal is due to oedema, collapse, and loss of myelinated fibres.

IV. Fascicular swelling.

V. Fatty atrophy and decreased bulk of the thenar muscles: This can be quantified by obtaining the ratio of signal intensity of the thenar muscles with respect to the hypothenar muscles. A value greater than one denotes fatty atrophy of the thenar muscles.

\section{Carpal tunnel syndrome in rheumatoid arthritis}

CTS is the most common compression neuropathy associated with rheumatoid arthritis [7], occurring in about $23 \%$ of patients with RA. It can be the initial manifesta- tion of the disease. However, in some cases the patients become accustomed to the paraesthesia due to its very gradual progression, and they may not describe typical CTS symptoms. Therefore, all patients with RA must be evaluated for CTS. The most common cause of compressive neuropathy in RA is flexor tenosynovitis (Figure 4). Other causes include severe wrist destruction with subluxation and following wrist arthrodesis for the treatment of RA.

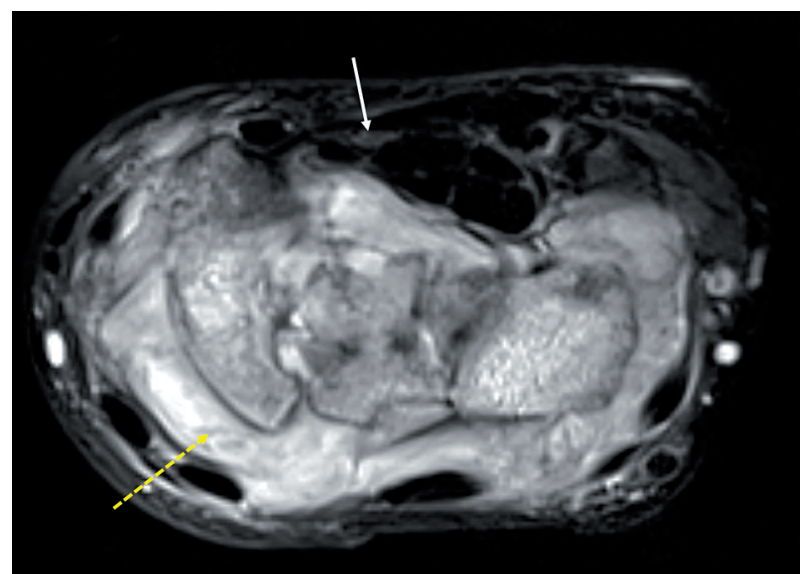

Figure 4. STIR axial image of the left wrist in a case of rheumatoid arthritis shows florid thickening of the synovium (dashed yellow arrow) and resultant flattening and angulation of the median nerve (white arrow) 

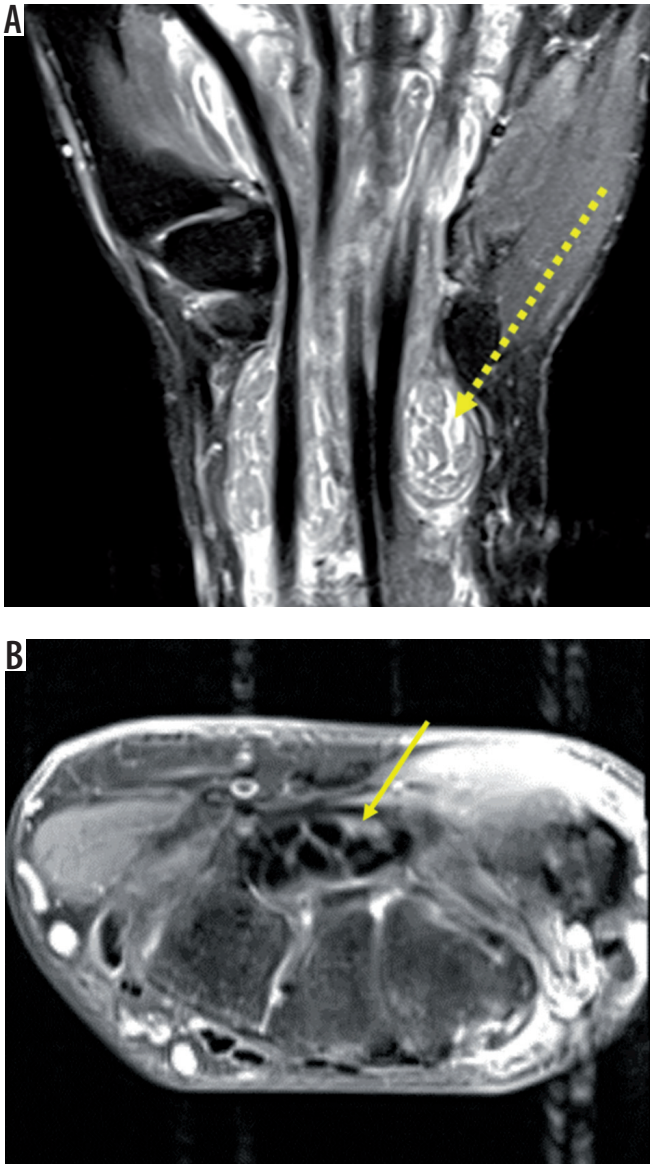

Figure 5. Coronal STIR (A) reveals gross distension of the flexor tendon sheaths with fluid and multiple round to elongated T1, T2 hypointense structures called rice-bodies within (dashed yellow arrow). T2 fat-saturated axial image (B) shows flattening and angulation of median nerve with intrasubstance hyperintensity (solid yellow arrow)

\section{Carpal tunnel syndrome in infections causing extrinsic nerve compression}

Compressive neuropathy of the median nerve in the carpal tunnel occurs due to interference in median nerve function from compression by inflammatory exudates in radial and ulnar bursae deep to the flexor retinaculum and due to flexor tenosynovitis (Figure 5). In cases of tubercular tenosynovitis, there can be development of rice bodies, which can further aggravate the compressive features. Rice bodies refer to oval to elongated similar-sized intraarticular bodies appearing hypointense on T1- and T2-weighted images. The common organisms leading to carpal tunnel syndrome include Mycobacterium tuberculosis, atypical mycobacterium, pyogenic bacteria such as staphylococcus and streptococcus species, sporotrichosis, coccidioidomycosis, and histoplasmosis.

\section{Carpal tunnel syndrome due to trauma}

Acute CTS is a rare compartment syndrome of the carpal tunnel that occurs after major trauma, typically distal radius fracture [8]. If median nerve compression is suspected
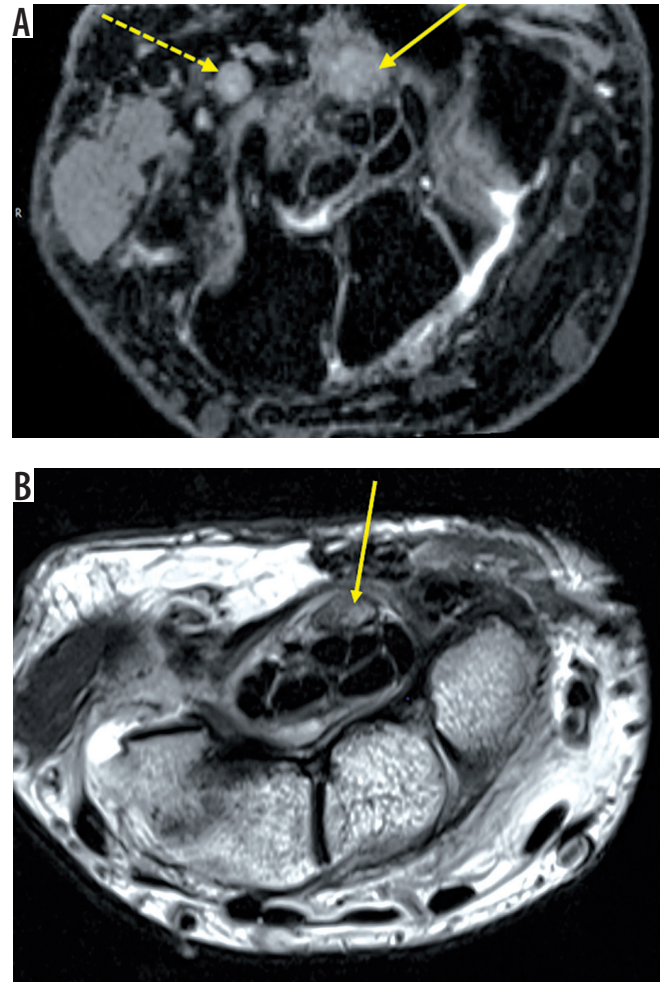

Figure 6. Axial DESS image (A) of the left wrist showing bulbous enlargement of the median nerve (solid yellow arrow) in the carpal tunnel and ulnar nerve in Guyon's canal (dashed yellow arrow) appearing heterogeneously hyperintense. Axial T2-weighted image (B) shows bulbous enlargement of median nerve with heterogeneously hyperintense signal pattern (solid yellow arrow). DESS coronal image (C) shows median nerve neuroma-in-continuity (solid yellow arrow)

on clinical examination with relevant clinical history of trauma, surgery must be performed to release the pressure. Imaging does not play a significant role in these cases.

Chronic CTS also develops following trauma due to degenerative arthritis that develops from an old fracture of the carpal bones (notably scaphoid) and malunited Colles' fracture.

\section{Post-traumatic neuroma}

A post-traumatic neuroma is a disorganised mass of fibroneural tissue that develops following trauma as a result of a failed attempt at nerve regeneration. Traumatic neuromas are high-grade injuries of the nerve and classified as i) end neuroma: as a result of complete transection, or ii) neuromain-continuity (NIC): as a result of partial nerve injury.

Neuromas are seen as bulbous enlargement of the nerve appearing heterogeneously hyperintense on $\mathrm{T} 2$ with heterogenous postcontrast enhancement (Figure 6). Heterogeneity on fluid-sensitive sequences are likely to represent a combination of fascicles, intralesional haemorrhage, and fibrosis.

Contrast material had been suggested as a plausible method to differentiate between neuroma and peripheral nerve sheath tumour (PNST), but it was found that most 

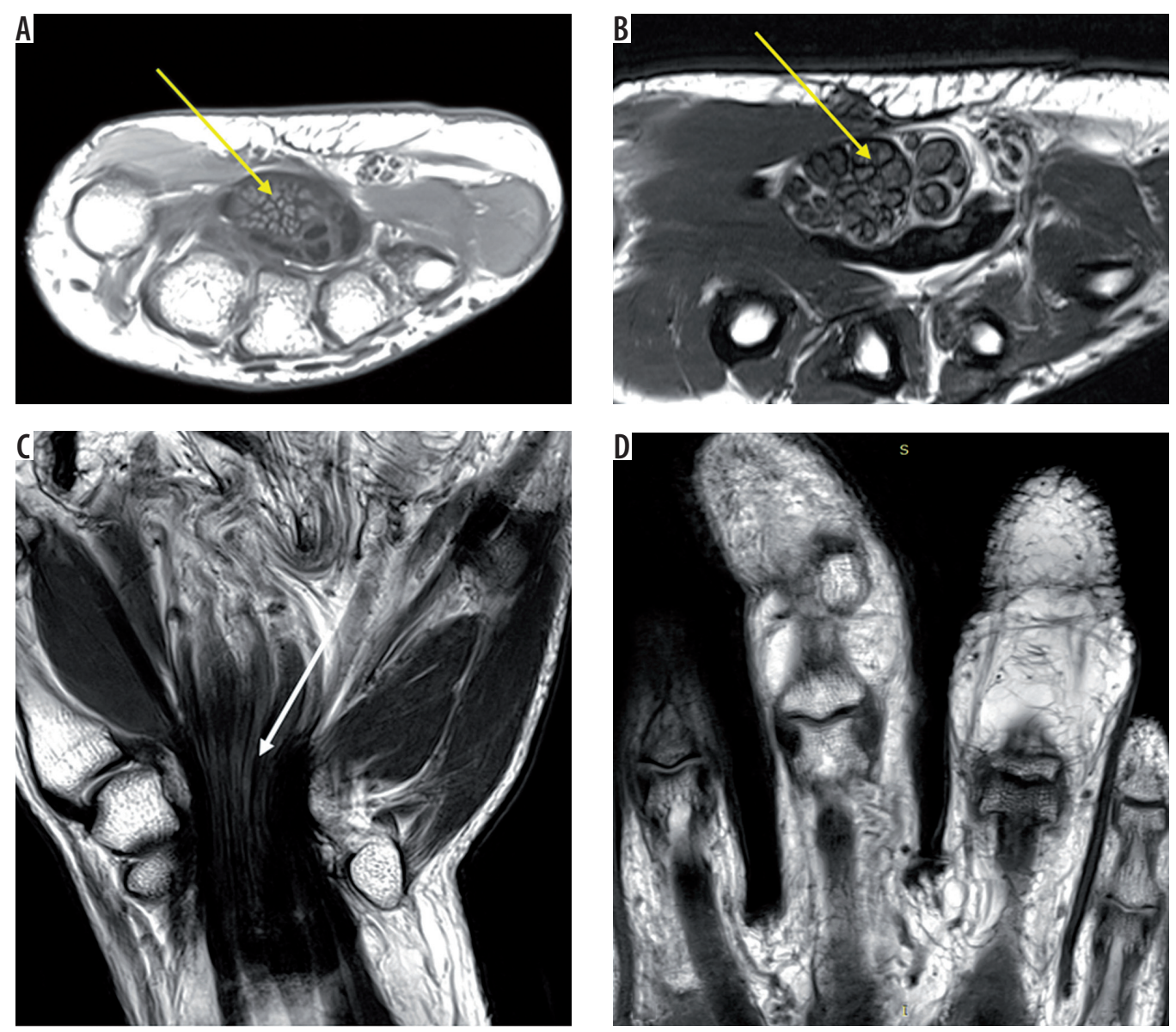

Figure 7. T1 axial (A) and T2 axial (B) images of the right wrist shows grossly thickened median nerve (solid yellow arrow) with thickened hypointense nerve fascicles surrounded and separated by hyperintense fibroadipose tissue, giving a coaxial cable appearance. Coronal T2-weighted image (C) shows 'spaghetti string' appearance of the lesion (white arrow). Coronal T1W image (D) shows marked lipomatous hypertrophy of the second and third digit

neuromas enhance post contrast administration, probably due to interruption in the peripheral nerve-blood barrier [9].

Neuromas and PNST can be differentiated by the presence of antecedent history of trauma 1-2 months prior and surrounding scarring and absence of 'target sign' seen classically in PNST [10].

\section{Fibrolipomatous hamartoma}

Fibrolipomatous hamartoma (FLH) refers to proliferation of mature adipocytes within peripheral nerves, separating axonal bundles in the perineurium [11]. The median nerve is the most frequently affected nerve, with involvement of other peripheral nerves less frequently. The patient generally presents with painless enlargement of the nerve and gradual-onset carpal tunnel syndrome.

The MRI findings of FLH are pathognomonic. It consists of markedly thickened nerve fascicles surrounded by evenly distributed high-intensity fibroadipose tissue giving a 'coaxial cable' appearance on axial images and 'spaghetti string' sign on coronal images (Figure 7). The fascicular pattern is well preserved [2]. The course of the nerve is altered due to its marked thickening. There is, however, no post-contrast enhancement seen in the affected nerve. In about two-thirds of patients with FLH, there is associated macrodystrophia lipomatosa along the distribution of the radial nerve seen radiographically as hypertrophy of the thumb and palm because there is overgrowth of all mesenchymal elements such as bones, fat, phalanges, tendons, nerves, and vessels.

FLH should be clinically, radiologically, and histologically differentiated from intraneural lipoma which occurs within the nerve sheath but do not involve nerve bundles.

\section{Hansen's neuritis}

Hansen's disease, or leprosy, is a non-fatal, slowly progressive chronic granulomatous infection caused by Mycobacterium leprae. The affected nerve shows nodular thickening and appears hyperintense on fat-saturated PD- and T2-weighted images [12] (Figure 8). There are multiple disruptions in the perineurium and epineurium with small variable sized outpouchings with loss of fascicular pattern. Alteration in the course of the nerve occurs due to nodular thickenings and outpouchings [2]. There is marked post-contrast enhancement.

These findings are, however, non-specific and can be seen in other hypertrophic neuropathies such as amyloid infiltration, chronic relapsing polyneuritis, Refsum's disease, and Gullain-Barre syndrome [13]. However, the pres- 

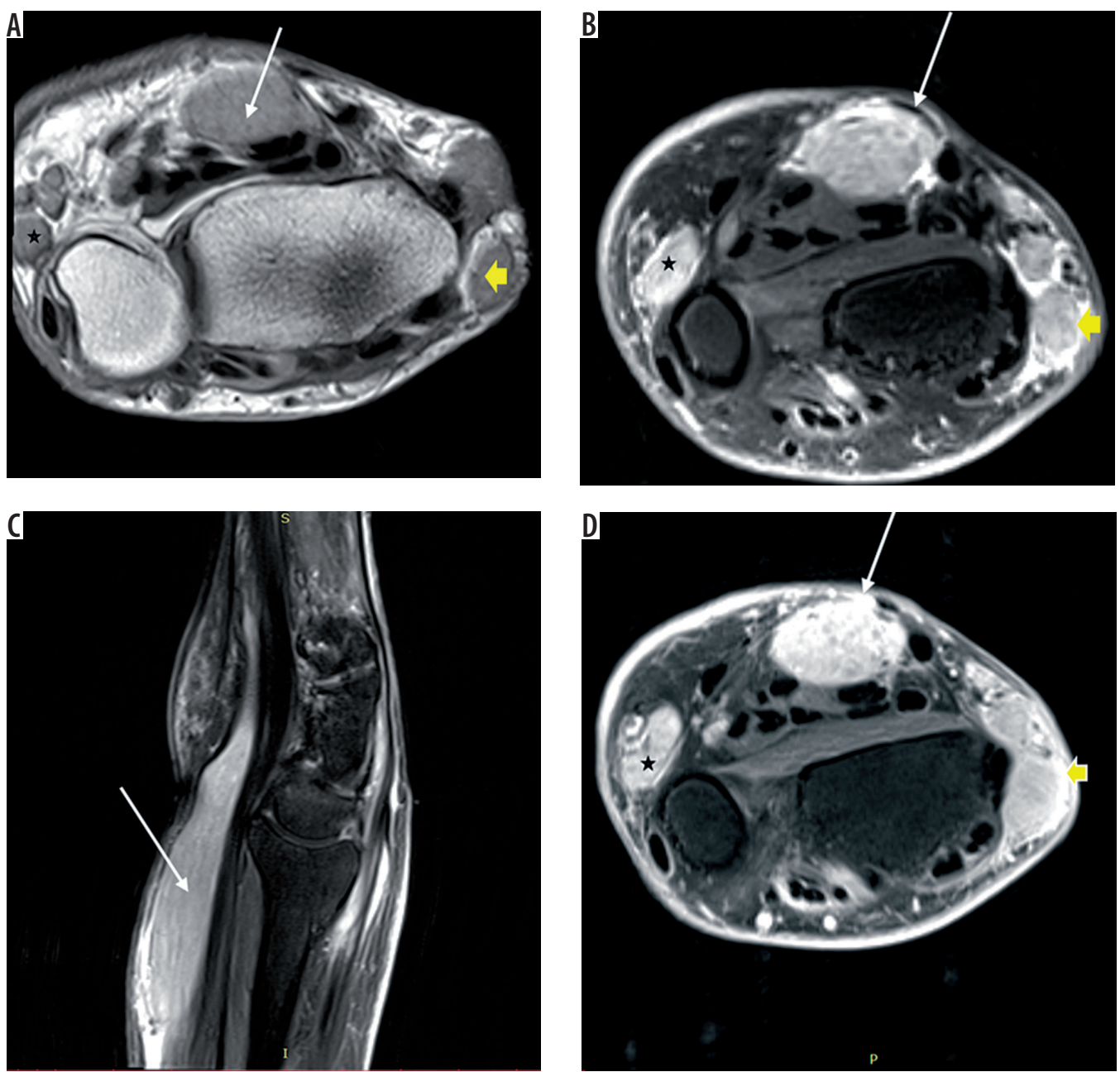

Figure 8. Axial T1 (A) and STIR (B) images of the right wrist reveal gross thickening of the median (white arrow), ulnar (black asterisk), and cutaneous branches of the radial nerve (short yellow arrow) showing altered signal intensity appearing hyperintense on T1-weighted and STIR sequences. STIR sagittal (C) images show the thickened hyperintense median nerve. Post-contrast axial image (D) shows intense heterogenous enhancement of the thickened nerves

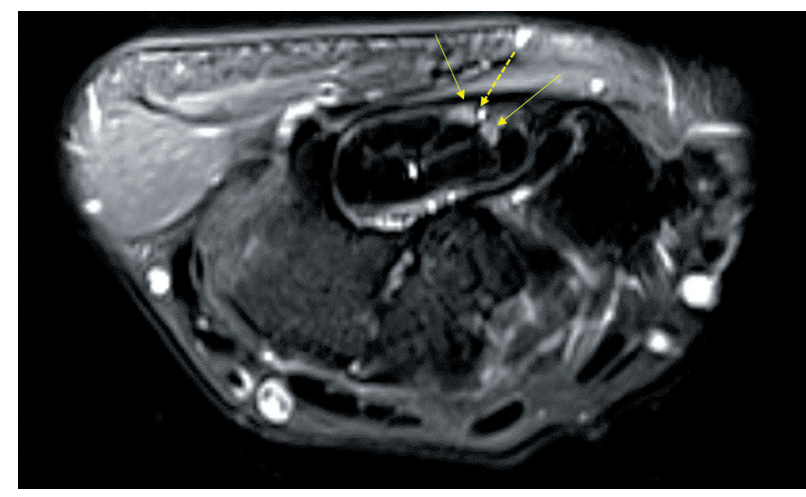

Figure 9. Axial T2 fat-saturated image of the left wrist shows bifurcation of median nerve (solid yellow arrow) within the carpal tunnel with a persistent median artery interposed between the nerve bundles (dashed yellow arrow)

ence of nodules or nerve sheath abscesses are specific to Hansen's disease [14]. The other specific findings include calcification of the nerve in the subacute and chronic phase of the disease [15].

MRI plays an important role in early detection of neuroarthropathic changes in asymptomatic patients, such as degradation and interruption of subcutaneous fat with effusion and synovitis in the affected joints [16].

\section{Bifid median nerve with persistent median artery}

Normally, the median nerve divides at the distal end of the carpal tunnel; therefore, any branching within or proximal to the carpal tunnel is considered a normal variant [17]. The bifurcation is associated with the presence of a vascular structure called persistent median artery, seen on axial T1- and T2-weighted images (Figure 9). A persistent median artery is recognised by its well-defined circular shape, its location close to the nerve, and higher signal intensity compared to the nerve. A bifid median nerve is predisposed to developing compressive neuropathy due to higher cross-sectional area compared to non-bifid median nerve.

\section{Conclusions}

MRI serves as an excellent modality for the evaluation of carpal tunnel and median nerve. It serves as an extremely 
valuable tool for the assessment of primary nerve pathologies as well as detection and characterisation of SOLs leading to compressive neuropathy. In a significant number of cases, MRI helps in the diagnosis of a nerve pathology even before the onset of functional changes.

\section{Disclosure}

The authors declare no conflicts of interest.

\section{References}

1. Polykandriotis E, Premm W, Horch RE. Carpal tunnel syndrome in young adults-an ultrasonographic and neurophysiological study. Minim Invasive Neurosurg 2007; 50: 328-334.

2. Thawait SK, Chaudhry V, Thawait GK, et al. High-resolution MR neurography of diffuse peripheral nerve lesions. AJNR Am J Neuroradiol 2011; 32: 1365-1372.

3. Chen YT, Williams L, Zak MJ, Fredericson M. Review of ultrasonography in the diagnosis of carpal tunnel syndrome and a proposed scanning protocol. J Ultrasound Med 2016; 35: 2311-2324.

4. Tsujii M, Hirata H, Morita A, Uchida A. Palmar bowing of the flexor retinaculum on wrist MRI correlates with subjective reports of pain in carpal tunnel syndrome. J Magn Reson Imaging 2009; 29: 1102-1105.

5. Klauser AS, Halpern EJ, De Zordo T, et al. Carpal tunnel syndrome assessment with US: value of additional cross-sectional area measurements of the median nerve in patients versus healthy volunteers. Radiology 2009; 250: 171-177.

6. Uchiyama S, Itsubo T, Yasutomi T, et al. Quantitative MRI of the wrist and nerve conduction studies in patients with idiopathic carpal tunnel syndrome. J Neurol Neurosurg Psychiatry 2005; 76: 1103-1108

7. Feldon P, Terrono AL. Carpal tunnel syndrome in rheumatoid arthritis. Techniques in Orthopaedics 2006; 21: 42-47.

8. Evans BT, Patel SP, Rozental TD. Carpal Tunnel Syndrome After Fractures and Other Trauma in Carpal Tunnel Syndrome and Related Median Neuropathies. Springer, Cham 2017; pp. 249-255.
9. Ahlawat S, Belzberg AJ, Montgomery EA, et al. MRI features of peripheral traumatic neuromas. Eur Radiol 2016; 26: 1204-1212.

10. Chhabra A, Williams EH, Wang KC, et al. MR neurography of neuromas related to nerve injury and entrapment with surgical correlation. AJR Am J Roentgenol 2010; 31: 1363-1368.

11. Toms AP, Anastakis D, Bleakney RR, et al. Lipofibromatous hamartoma of the upper extremity: a review of the radiologic findings for 15 patients. AJR Am J Roentgenol 2006; 186: 805-811.

12. Sen D, Satija L, Chatterji S, et al. Ultrasonography and magnetic resonance imaging of ulnar nerve abscess in leprosy. Med J Armed Forces India 2016; 72: 78 .

13. Kulkarni M, Chauhan V, Bharucha M, et al. MRI imaging of ulnar leprosy abscess. J Assoc Physicians India 2009; 57: 175-176.

14. Hari S, Subramanian S, Sharma R. Magnetic resonance imaging of ulnar nerve abscess in leprosy: a case report. Lepr Rev 2007; 78: 155-159.

15. Martinoli C, Airaldi S, Zaottini F. Ultrasound of the peripheral nerves. In: Musculoskeletal Imaging Volume 2: Metabolic, Infectious, and Congenital Diseases; Internal Derangement of the Joints; and Arthrography and Ultrasound. Taljanovic MS, Omar IM, Hoover KB, Chadaz TS. Oxford University Press, Oxford 2019; p. 382.

16. Luyckx G, Vanhoenacker FM, Parizel PM. Exotic pathology of the hand and foot. A pictorial review. JBR-BTR 2008; 91: 160-165.

17. Pierre-Jerome C, Smitson RD, Shah RK, et al. MRI of the median nerve and median artery in the carpal tunnel: prevalence of their anatomical variations and clinical significance. Surg Radiol Anat 2010; 32: 315-322. 